\title{
Interventioneller Verschluss oder konservative Therapie?
}

Fragestellung: Ist der interventionelle Verschluss eines offenen Foramen ovale bei Patienten mit kryptogenem Schlaganfall im Alter unter 60 Jahren einer medikamentösen Therapie mit Thrombozytenfunktionshemmern oder oralen Antikoagulanzien überlegen?

Hintergrund: Bei Patienten mit kryptogenem Schlaganfall im Alter unter 60 Jahren wird in Fall-Kontroll-Studien überproportional häufig ein offenes Foramen ovale gefunden. Daher wurde angenommen, dass ein kausaler Zusammenhang zwischen dem offenen Foramen ovale und kryptogenen Schlaganfällen besteht. Die Mitte letzten Jahres publizierte CLOSUREI-Studie hatte allerdings bei Verwendung des STARFlex-Verschlusssystems keine Überlegenheit der Intervention gegenüber einer konservativen Therapie finden können. In der vorliegenden RESPECT-Studie sollte der Occluder der Firma Amplatzer untersucht werden.

Patienten und Methodik: Bei dieser Untersuchung handelt es sich um eine prospektive multizentrische randomisierte Studie, bei Patienten im Alter unter 60 Jahren mit kryptogenem Schlaganfall. Die Studienteilnehmer wurden entweder in eine Interventionsgruppe mit Verschluss des offenen Foramen ovale oder in eine Gruppe mit konservativer Therapie mit Thrombozytenfunktionshemmern oder oralen Antikoagulanzien randomisiert. Der primäre Studienendpunkt war die Kombination aus erneutem nicht tödlichem ischämischem Schlaganfall, tödlichem ischämischem Insult oder Tod innerhalb von 30 Tagen nach der Implantation beziehungsweise 45 Tagen nach der Randomisierung.

Ergebnisse: In die Studie wurden 980 Patientin in einem mittleren Alter von 46 Jahren eingeschlossen. Die meisten Patienten hatten ein großes offenes Foramen ovale und 35\% der Patienten wiesen ein Vorhofseptumaneurysma auf. Die mediane Zeit vom initialen Schlaganfall bis zur Randomisierung betrug 120 Tage. Die durchschnittliche Beobachtungszeit innerhalb der Studie lag bei 2,6 Jahren. Die Drop-out-Rate betrug 14,2\% in der konservativ behandelten Gruppe und 9,2\% in der interventionell behandelten Gruppe. Die konservative Behandlung bestand aus einer Monotherapie mit Acetylsalicylsäure bei 46,5\% der Patienten, einer Monotherapie mit Warfarin bei 25,2\% der Patienten, einer Monotherapie mit Clopidogrel bei 14\% der Patien-

Carroll JD, Saver JL, Thaler DE et al; RESPECT Investigators. Closure of patent foramen ovale versus medical therapy after cryptogenic stroke. N Engl J Med 2013; 368: 1092-100 ten und der Kombination von Acetylsalicylsäure plus Dipyridamol bei $8 \%$ der Patienten.

Insgesamt tritt in dieser Studie bei 25 Patienten ein primärer Endpunkt ein. Dabei handelte es sich in allen
Fällen um nicht tödliche ischämische Schlaganfälle. In der Intention-to-treat-Analyse traten neun Ereignisse bei den Patienten mit Verschluss des offenen Formen ovale auf und 16 in der konservativ behandelten Gruppe. Dies entspricht einer Risikoreduktion von $51 \%$, die statistisch nicht signifikant war. In der Per-Protocol-Analyse traten 20 primäre Endpunkte auf, davon sechs in der Verschlussgruppe und 14 in der konservativ behandelten Gruppe. Die hier beobachtete Risikoreduktion von $63 \%$ war statistisch signifikant.

Schwerwiegende unerwünschte Ereignisse traten bei $23 \%$ der Patienten der Verschlussgruppe und bei 21,6\% der Patienten der konservativ behandelten Gruppe auf. Dieser Unterschied war statistisch nicht signifikant. Nebenwirkungen der interventionellen Therapie traten bei 21 von 499 Patienten auf. Dies entspricht $4,2 \%$.

Schlussfolgerung: Der Verschluss eines offenen Foramen ovale bei Patienten mit kryptogenem Schlaganfall mit dem AMPLATZER-Device hat einen starken Trend zugunsten der interventionellen Therapie bezüglich rezidivierenden ischämischen Insulten. Der Unterschied war allerdings statistisch nicht signifikant.

\section{- Kommentar von Hans-Christoph Diener, Essen}

\section{Antikoagulation ebenso wirksam}

Diese große prospektive randomisierte Studie ist methodisch und technisch gut durchgeführt. Allerdings war sie bezüglich des primären Endpunktes in der Intention-to-treat-Analyse nicht signifikant. Der Unterschied zwischen der Intention-totreat- und der Per-Protocol-Analyse liegt darin, dass einige der Patienten, die in die konservative Therapiegruppe randomisiert worden waren, einen Verschluss des offenen Foramen ovale erhielten. Betrachtet man die Subgruppenanalysen, dann hat es den Anschein, als wenn Foramina mit großem Shunt-Volumen und Vorhofseptumaneurysma am ehesten von einem Verschluss profitieren. Betrachtet man die konservative Therapie, ergab sich bei antikoagulierten Patienten kein Unterschied zur Intervention, während es einen signifikanten Unterschied für Patienten gab, die nur mit Thrombozytenfunktionshemmern behandelt wurden. Fasst man die Datenlage zusammen, ist leider immer noch nicht endgültig geklärt, ob der Verschluss eines offenen Foramen ovale beim kryptogenen Schlaganfall wirklich weitere Schlaganfälle verhindert. Nach den Ergebnissen der RESPECT-Studie ist eine orale Antikoagulation offenbar genauso wirksam. Der Verschluss des offenen Foramen ovale käme dann insbesondere bei Patienten zum Einsatz, bei denen unter oraler Antikoagulation ein Rezidivereignis eintritt. 